\title{
Comparative Outcomes of Commonly Used Off-Label Atypical Antipsychotics in the Treatment of Dementia-Related Psychosis: A Network Meta-analysis
}

\author{
Ismaeel Yunusa · Nazia Rashid · George N. Demos · Bhargavi S. Mahadik • \\ Victor C. Abler · Krithika Rajagopalan
}

Received: November 20, 2021 / Accepted: February 3, 2022 / Published online: March 5, 2022

(C) The Author(s) 2022

\section{ABSTRACT}

Introduction: Dementia-related psychosis (DRP) is characterized by hallucinations and delusions, which may increase the debilitating effects of underlying dementia. This network meta-analysis (NMA) evaluated the comparative efficacy, safety, and acceptability of atypical antipsychotics (AAPs) commonly used off label to treat DRP.

Methods: We included 22 eligible studies from a systematic literature review of AAPs (quetiapine, risperidone, olanzapine, aripiprazole, and brexpiprazole) used off label to treat DRP. Study outcomes were: (1) efficacy-neuropsychiatric inventory-nursing home (NPI-NH psychosis subscale), (2) safety-mortality, cerebrovascular events (CVAEs), and others (somnolence, falls, fractures, injuries, etc.), and (3) acceptabilitydiscontinuations due to all causes, lack of

Supplementary Information The online version contains supplementary material available at https:// doi.org/10.1007/s12325-022-02075-8.

I. Yunusa

College of Pharmacy, University of South Carolina, Columbia, SC, USA

N. Rashid · G. N. Demos · V. C. Abler

Acadia Pharmaceuticals, Inc, San Diego, CA, USA

B. S. Mahadik $(\square) \cdot$ K. Rajagopalan

Anlitiks Inc., Dover, MA, USA

e-mail: b.mahadik@anlitiks.com efficacy, and adverse events (AEs). We used random-effects modeling to estimate pooled standardized mean differences (SMDs) for NPI$\mathrm{NH}$ psychosis subscale scores and odds ratios (OR) for other dichotomous outcomes, with their respective 95\% confidence intervals (CIs). Results: Compared with placebo, aripiprazole (SMD - 0.12; 95\% CI - 0.31, 0.06), and olanzapine (SMD - 0.17; 95\% CI - 0.04; 0.02) demonstrated small, non-significant numerical improvements in NPI-NH psychosis scores (5 studies; $n=1891$ ), while quetiapine (SMD 0.04; 95\% CI $-0.23,0.32$ ) did not improve symptoms. The odds of mortality (15 studies, $n=4989$ ) were higher for aripiprazole (OR 1.58; 95\% CI 0.62, 4.04), brexpiprazole (OR 2.22; 95\% CI $0.30,16.56$ ), olanzapine (OR 2.21; 95\% CI $0.84,5.85$ ), quetiapine (OR $1.68 ; 95 \%$ CI 0.70 , 4.03), and risperidone (OR 1.63; 95\% CI 0.93, 2.85) than for placebo. Risperidone (OR 3.68; 95\% CI 1.68, 8.95) and olanzapine (OR 4.47; $95 \%$ CI 1.36, 14.69) demonstrated significantly greater odds of CVAEs compared to placebo. Compared with placebo, odds of all-cause discontinuation were significantly lower for aripiprazole (OR 0.71; 95\% CI 0.51, 0.98; 20 studies; 5744 patients) and higher for other AAPs. Aripiprazole (OR $0.5 ; 95 \%$ CI $0.31,0.82$ ) and olanzapine (OR 0.48 ; 95\% CI 0.31, 0.74) had significantly lower odds of discontinuation due to lack of efficacy (OR 12 studies; $n=4382$ ) compared to placebo, while results for quetiapine and risperidone were not significant. 
Compared with placebo, the odds of discontinuation due to AEs (19 studies, $n=5445$ ) were higher for olanzapine (OR 2.62; 95\% CI 1.75, 3.92), brexpiprazole (OR 1.80; 95\% CI 0.80, 4.07), quetiapine (OR 1.25 ; 95\% CI 0.82, 1.91), aripiprazole (OR 1.38; 95\% CI 0.90, 2.13), and risperidone (OR 1.41; 95\% CI 1.02, 1.94).

Conclusions: Overall results demonstrate that, compared with placebo, quetiapine is not associated with improvement in psychosis in patients with dementia, while olanzapine and aripiprazole have non-significant small numerical improvements. These off-label AAPs (quetiapine, risperidone, olanzapine, aripiprazole, and brexpiprazole) are associated with greater odds of mortality, CVAEs, and discontinuations due to AEs than placebo. These results underscore the ongoing unmet need for newer pharmacological options with a more favorable benefit-risk profile for the treatment of DRP.

Keywords: Dementia-related Hallucination; Delusions; antipsychotics; Network meta-analysis; Efficacy; Safety; Acceptability

\section{Key Summary Points}

All atypical antipsychotics used off label to treat dementia-related psychosis included in the analysis were associated with greater odds of mortality, cerebrovascular adverse events, and discontinuations due to adverse events compared with placebo

Compared to placebo, olanzapine, and aripiprazole demonstrated a small, nonsignificant improvement in symptoms of psychosis

Although olanzapine improved psychosis symptoms, it was associated with a greater risk of mortality and cerebrovascular adverse events than placebo

\section{INTRODUCTION}

Dementia is a progressive, disabling condition which causes a decline in the cognitive and functional abilities [1]. Currently, there are about 50 million people living with dementia worldwide and about 7.9 million in the US [2]. This number is expected to increase substantially in the coming years given the rise in the geriatric population and increasing life expectancy. The most common types of dementia include Alzheimer's disease (AD) dementia, vascular dementia $(\mathrm{VaD})$, dementia with Lewy bodies (DLB), frontotemporal dementia (FTD), and Parkinson disease (PD) dementia. Dementia patients suffer from a host of neuropsychiatric symptoms (NPS) such as depression, anxiety, aggression, and agitation as well as delusions and hallucinations, a characteristic hallmark of dementia-related psychosis (DRP) [3]. It is estimated that $20-70 \%$ of dementia patients suffer from DRP [4]. While delusions can cause anxiety and fear that can adversely impact the patient's daily activities, communication, and patient relationships, hallucinations can result in limited participation in social activities. This results in a decline in the quality of life of patients, increased caregiver burden, and increased hospital stays, which consequently increase the chances of early institution placements [5].

To date, no FDA-approved pharmacological treatment exists for the treatment of NPS, including symptoms of DRP. However, AAPs have been prescribed off label for treating NPS in about a third of patients with dementia [6]. While AAPs such as quetiapine, risperidone, olanzapine, aripiprazole, and brexpiprazole are commonly used in patients with dementia, clinical evidence suggests that they exhibit only modest NPS improvements while imposing a significant safety liability in this vulnerable population. Additionally, research 
suggests that patients treated with AAPs have an elevated risk of mortality and cerebrovascular adverse events (CVAEs); the risk of mortality may increase with their longer duration of use [7]. In recognition of the safety risks associated with these AAPs, the FDA issued a boxed warning about the increased risk of mortality [7]. In addition to increased risk of mortality, AAPs may also impose high treatment-emergent adverse effect burden related to weight gain, lipid abnormalities, glucose abnormalities, prolactin elevation, sedation, extrapyramidal symptoms (EPS), gait disturbance, and parkinsonism, among others $[8,9]$.

While limited randomized head-to-head clinical trials of these AAPs have demonstrated comparative efficacy, safety, and acceptability in treating NPS on a broad range of outcomes, previous pairwise and network meta-analyses suggest that no AAP is more beneficial or safe than another in treating NPS [10, 11]. Two separately conducted network meta-analyses (NMA) that examined the role of AAPs in dementia (Yunusa 2019, Watt 2017) [10, 11] have confirmed similar findings. Specifically, Yunusa et al. found that AAPs showed numerical, non-significant improvements in NPS compared to placebo; however, they were significantly associated with higher risks of CVAEs and death. Surprisingly, no NMAs have evaluated the comparative acceptability of AAPs on a broad range of outcomes related to discontinuations due to all causes, lack of efficacy, and adverse events in patients with DRP. Given this void in the literature and the increasing focus on the appropriate use of AAPs in patients with DRP, it is crucial to quantitatively estimate the comparative efficacy, safety, and acceptability of AAPs in the treatment of DRP. Such research may also be well served by an NMA using data from a comprehensive systematic literature review (SLR) that includes non-randomized observational studies and open-label trials. Thus, the objective of this study was to estimate the comparative efficacy, safety, and acceptability of AAPs for the treatment and management of DRP through an NMA.

\section{METHODS}

\section{Study Selection and Data Extraction}

We conducted this NMA using data from a SLR [12]. The SLR followed the preferred reporting items for systematic reviews and meta-analyses (PRISMA) statement [13], aligned with the Centre for Reviews and Dissemination guide for conducting systematic reviews [14], with searches from January 2000 up to March 2021 (Fig. 1). The searches identified randomized controlled trials (RCTs), open-label trials, and observational studies of atypical antipsychotic monotherapy in the DRP population. Trials included patients ( $\geq 40$ years of age, those living in the community or nursing home) with dementia of the different subtypes $(\mathrm{AD}, \mathrm{VaD}$, FTD, DLB, PD, or mixed dementia) who were diagnosed with symptoms of DRP. Searches were performed in MEDLINE/PubMed, PsycINFO, EMBASE, and Cochrane Central Register of Controlled Trials. Only studies published in English were included. All abstracts were initially reviewed for eligibility, and full texts were reviewed only for relevant studies by two individual researchers. Discrepancies about including studies were resolved by consultation with a senior reviewer. All selected studies were assessed for quality using the Cochrane Collaboration's tool for assessing risk of bias [15] for randomized trials to utilize low, unclear, or high risk of bias using seven domains (namely sequence generation, allocation concealment, blinding of participants and personnel, blinding of outcome assessment, incomplete outcome data, selective outcome reporting, and 'other sources of bias'), while non-randomized observational studies were assessed using the Newcastle-Ottawa scale by two independent researchers, results for which have been reported elsewhere [16].

\section{Outcome Measures}

The efficacy outcome measure was the Neuropsychiatric Inventory-Nursing Home (NPH$\mathrm{NH}$ ) version psychosis sub-scale score (sum of 


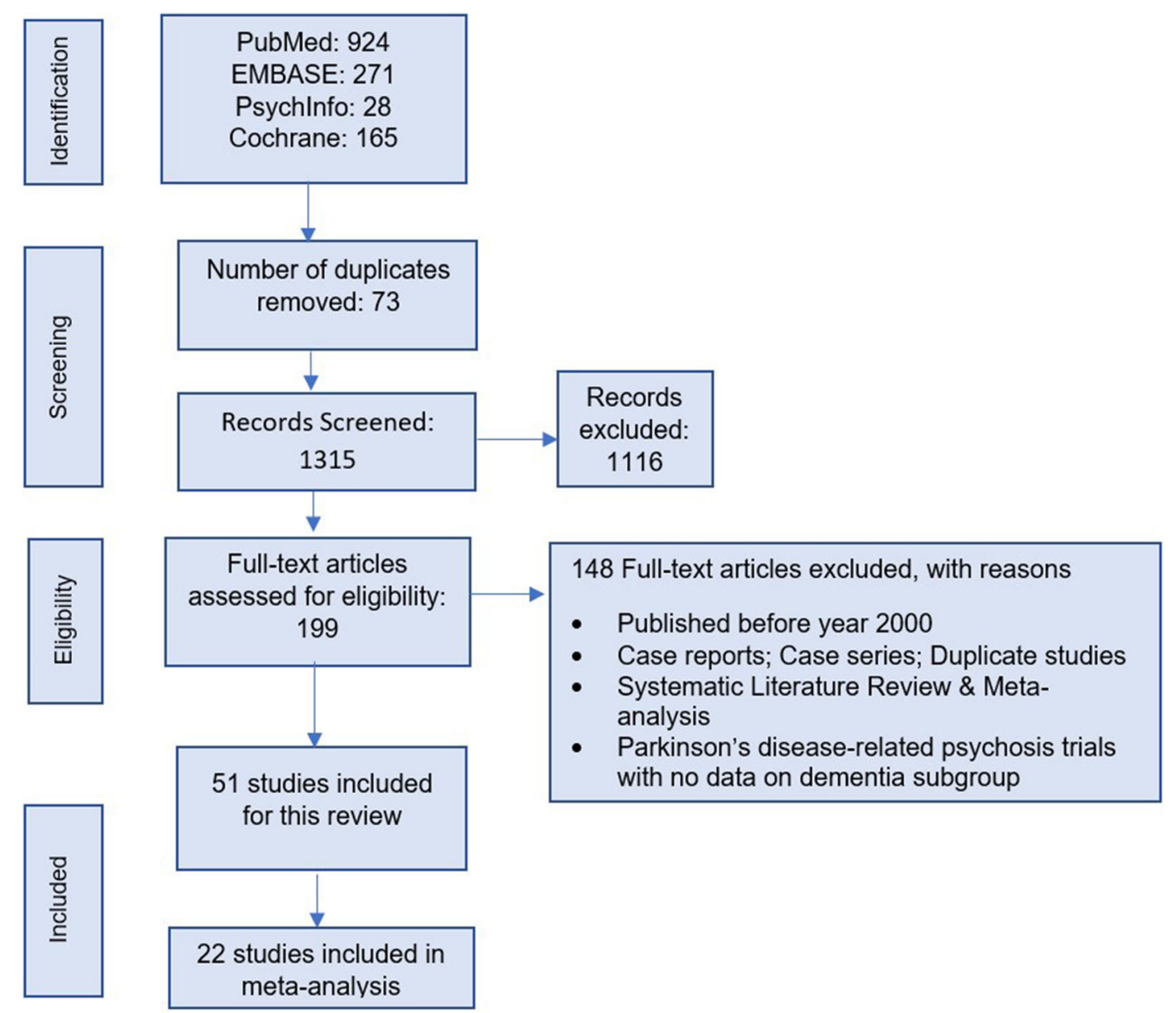

Fig. 1 PRISMA study selection flowchart

hallucinations and delusions sub-scores). The safety outcomes included all-cause mortality, CVAEs (measured as of stroke or transient ischemic attacks), somnolence, and falls, fractures, and injuries (FFI). This NMA also allowed for the comparative assessment of real-world effectiveness related to real-world "acceptability" among patients that is measured as discontinuations due to (1) all causes, (2) lack of efficacy, and (3) adverse events. Additionally secondary outcomes that were assessed included mini mental state examination (MMSE) and cognitive impairment.

\section{Network Meta-analysis and Data Synthesis}

This NMA followed the PRISMA Extension Statement for Reporting of Systematic Reviews
Incorporating Network Meta-analyses of Health Care Interventions (PRISMA-NMA) [17]. The NMA included studies of AAP treatment for DRP in the elderly and was conducted using the multivariate meta-analysis approach using the Network meta package in Stata statistical software (Stata version 15.1) [18, 19]. Transitivity (i.e., distribution of patient and study characteristics that are potential modifiers of treatment effects and are sufficiently similar in different sets of trials before an indirect comparison) is a fundamental assumption underlying NMA [20]. Transitivity was analyzed by qualitatively assessing the distribution of the potential effect modifiers across the different direct comparisons in our data [21], and we assumed transitivity after studying the population characteristics. All analyses were conducted 
by the lead author. All participants were diagnosed with DRP with a mean age of 80.3 years; $>$ $50 \%$ were females. All outcomes were analyzed using random effects models wherever possible. Instances where random effects model was not a good fit, fixed effect model was used to analyze the outcomes. Loop-specific approach assuming loop-specific heterogeneity was used to evaluate consistency by examining the agreement between direct and indirect treatment effects for relevant outcomes [22-24]. The $p$-value for inconsistency was 0.1 [22]. As inconsistency was reported in a few outcomes, a design by interaction inconsistency model was fitted wherever necessary and reported in the list of supplementary material (Supplementary Table 5) [25]. For treatments that contrast in the network, presence of inconsistency was evaluated using the node-splitting analysis method to assess agreement between direct and indirect evidence on a specific node $[26,27]$.

To ensure homogeneity of results for efficacy outcomes, the NMA was based on a comparable follow-up period for the outcome or time points of each trial as reported. Results for continuous outcomes were reported as mean change from baseline scores, standard deviation, and sample size in each arm. Results for categorical outcomes were reported as proportion of events recorded and sample size in every arm. Results for continuous outcomes were expressed as the standard mean difference (SMD), based on changes from baseline to the final endpoint, with their associated confidence intervals (95\% CIs). The clinical significance of these SMDs can be interpreted as small, medium, and large using Cohen's rule of thumb (e.g., SMD $=0.2$, low; $\mathrm{SMD}=0.5$, medium; $\mathrm{SMD}=0.8$, large) [28]. Since higher scores on the MMSE scale meant lower level of cognitive impairment, the algebraic signs for individual scores were reversed before analysis. Therefore, lower SMDs were equivalent to improvements across all continuous outcomes. Results for binary or dichotomous outcomes were expressed as odds ratios (ORs) and their respective 95\% CIs. Surface under cumulative ranking curve (SUCRA) was used to rank treatments. SUCRA represents the predicted percentage of efficacy, safety, or acceptability for every treatment option to rank the most favorable compared to a hypothetical treatment option that will certainly rank the best $[29,30]$. SUCRA is a numeric representation of an outcome's overall treatment ranking that assigns a single number to each therapy based on values ranging from 0 to $100 \%$. Along with SMD and OR estimates, SUCRA was used to ascertain treatment ranking where the closer a treatment's SUCRA value was to $100 \%$, the more likely it would be the more acceptable or safe alternative; the lower and closer the SUCRA value was to $0 \%$, the less likely it would be the most acceptable or safe alternative. This article is based on previously conducted studies and does not contain any new studies with human participants or animals performed by any of the authors.

\section{RESULTS}

\section{Included Trials and Patient Characteristics}

The initial SLR [12] search strategy included all AAPs to ensure a comprehensive search. Upon screening articles for eligibility, a total of 51 full-text studies of quetiapine, risperidone, aripiprazole, brexpiprazole, olanzapine, and ziprasidone contributed to the SLR's qualitative synthesis. This NMA included all AAPs from studies with comparative quantitative data on the outcomes of interest. A total of 22 [7, 31-51] published studies (18 double-blinded RCTs, 1 rater blinded RCT, 2 open-label studies and 1 observational study) were included in the NMA. The PRISMA diagram depicts the study selection process (Fig. 1). Thirteen studies were conducted in a nursing home or institutionalized setting, one study each in an outpatient setting and hospital setting, and seven studies in a mixed setting (i.e., nursing home, inpatient or outpatient setting). The average study duration in the included trials was 13 weeks with a median of 10 weeks and range of 6-48 weeks, and the mean age was $80.1 \pm 3.6$ years among the total number of patients $(n=5971)$. Further details of the study designs and baseline characteristics from the included trials are presented in Supplementary Table 1. The baseline NPI-NH 


\section{NPI-NH Psychosis Subscale}

Drug
Aripiprazole vs. Placebo
Olanzapine
Quetiapine

Fig. 2 Forest plot for efficacy outcome of atypical antipsychotics in comparison with placebo. Forest plot for NPI-NH psychosis subscale, representing pooled standard mean differences comparing each treatment

psychosis score appeared similar across all the included studies ranging from 6.1 to 10.75 .

\section{Outcomes}

\section{Efficacy Outcomes}

NPI-NH Psychosis Subscale Supplementary Figure 1 depicts the network diagram and Fig. 2 depicts the forest plot for the NPI-NH psychosis subscale. Of the five studies [34, 43, 47, 48, 51] ( $n=1891$ patients) included for analysis, aripiprazole (SMD - 0.12; 95\% CI -0.31 to 0.06 ) and olanzapine (SMD $-0.17 ; 95 \% \mathrm{CI}-0.04$ to 0.02 ) demonstrated small numerical improvements in NPI-NH psychosis subscale scores, while quetiapine (SMD 0.04; 95\% CI -0.23 to 0.32) did not improve psychosis symptoms. Results for all AAPs compared to each other were not statistically significant (Supplementary Table 2). SUCRA results suggest that olanzapine $(81.4 \%)$ had the highest probability of improving psychosis based on the NPI-NH psychosis subscale.

\section{Safety Outcomes}

Primary Safety Outcomes (Mortality, CVAEs, Somnolence, Falls, Fractures, and Injuries) Supplementary Figure 1 depicts the network diagram, and Fig. 3 depicts the forest plots for primary safety outcomes. For the analysis of mortality, a total of 15 studies $[31-36,39,40,42,43,46,48,50,51](n=4989$ patients) demonstrate that the odds of mortality were numerically higher for brexpiprazole
(OR 2.22; 95\% CI 0.30, 16.66), olanzapine (OR 2.21; 95\% CI 0.84, 5.85), aripiprazole (OR 1.58; $95 \%$ CI $0.62,4.04$ ), quetiapine (OR 1.68; 95\% CI $0.70,4.03$ ), and risperidone (OR 1.63; 95\% CI $0.93,2.85)$ compared to placebo. SUCRA results show that placebo $(87.4 \%)$ had the highest probability of being the safest for mortality.

For CVAEs, a total of 12 studies $[32,33,35,36,41-43,46,48-51] \quad(n=3414)$ showed that the odds of CVAEs were significantly higher for olanzapine (OR 4.47; 95\% CI $1.35,14.69$ ) and risperidone (OR 3.88; 95\% CI $1.68,8.95)$ than for placebo. SUCRA results show that placebo $(87.72 \%)$ had the highest probability of being the safest for CVAEs.

For somnolence, a total of 13 studies [32, 35, 38-40, 42-44, 46-48, 50, 51] $(n=3853$ patients) show that, compared to placebo, the odds of somnolence were significantly higher for aripiprazole (OR 2.73; 95\% CI 1.20, 6.18), olanzapine (OR 3.79; 95\% CI 2.17, 6.62), quetiapine (OR 6.65; 95\% CI 2.76, 16.02), and risperidone (OR 2.44; 95\% CI 1.72, 3.47). The odds of somnolence were comparatively lower for brexpiprazole (OR 1.70; 95\% CI 0.52, 5.61) compared to placebo; however, the results were non-significant. SUCRA results show that placebo (96.4\%) had the highest probability of being the safest for somnolence.

For the analysis of falls, fractures, and injuries, a total of 15 studies $[32,33,35,36,39,40,42,43,45-51](n=4509$ patients) demonstrate that the odds of falls, fractures, and injuries were lowest for 

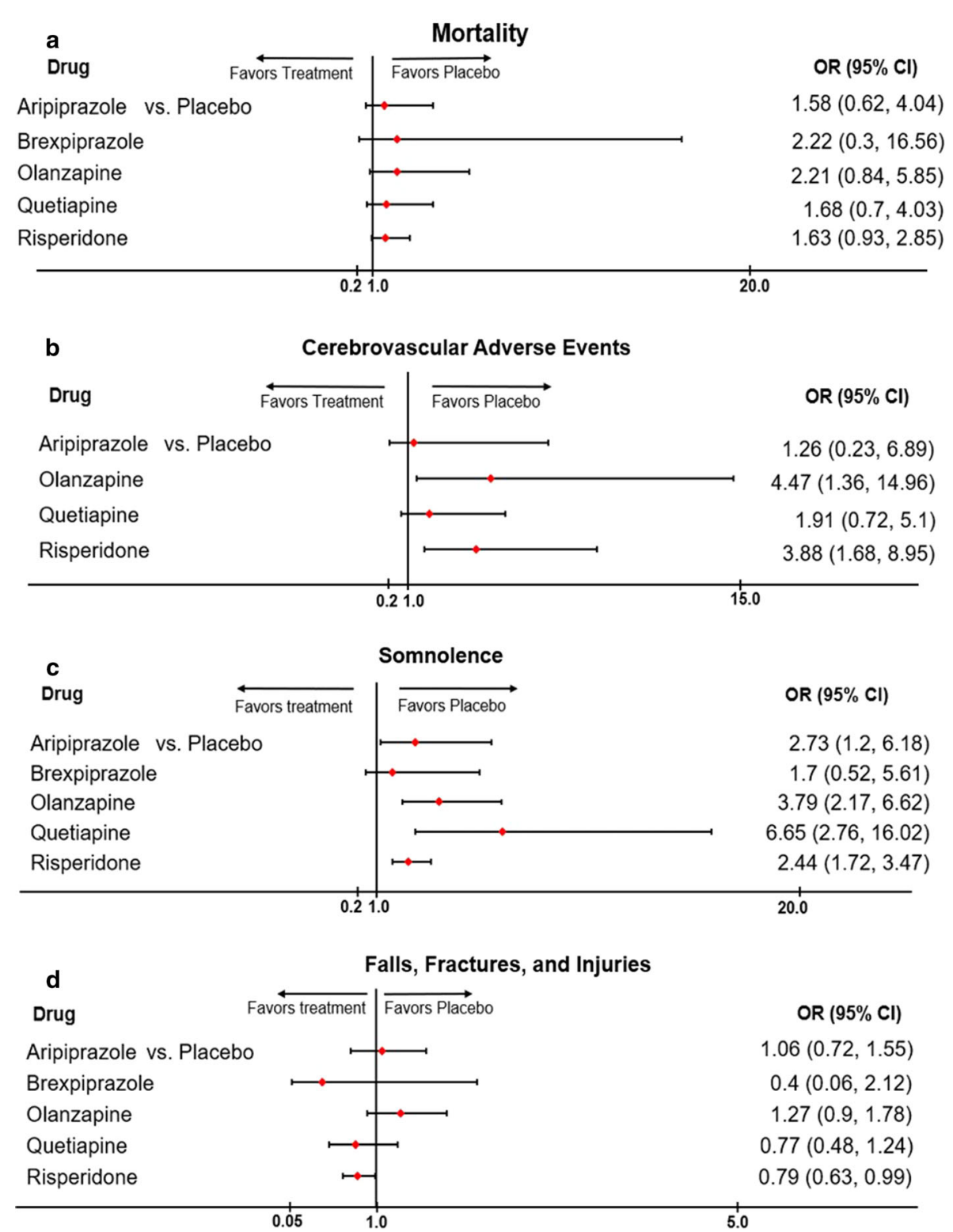

Fig. 3 Forest plots for primary safety outcomes of a typical antipsychotics compared with placebo. a Forest plot for mortality, representing pooled odds ratio comparing each treatment option with placebo. The diamonds denote the point estimate for each treatment compared to placebo, while the horizontal line denoted the $95 \%$ CI. If the $95 \%$ CI does not cross 1 (null value); the results imply statistical significance. b Forest plot for CVAE, representing pooled odds ratio comparing each treatment option with placebo. The diamonds denote the point estimate for each treatment compared to placebo, while the horizontal line denoted the 95\% CI. If the 95\% CI does not cross 1 (null value); the results imply statistical significance. c Forest plot for somnolence, representing pooled odds ratio comparing each treatment option with placebo. The diamonds denote the point estimate for each treatment compared to placebo, while the horizontal line denoted the $95 \%$ CI. If the $95 \%$ CI does not cross 1 (null value); the results imply statistical significance. d Forest plot for falls, fractures, and injuries, representing pooled odds ratio comparing each treatment option with placebo. The diamonds denote the point estimate for each treatment compared to placebo, while the horizontal line denoted the $95 \%$ CI. If the $95 \%$ CI does not cross 1 (null value); the results imply statistical significance 
a

Drug
Aripiprazole vs. Placebo
Brexpiprazole
Olanzapine
Quetiapine
Risperidone

All-Cause Discontinuation

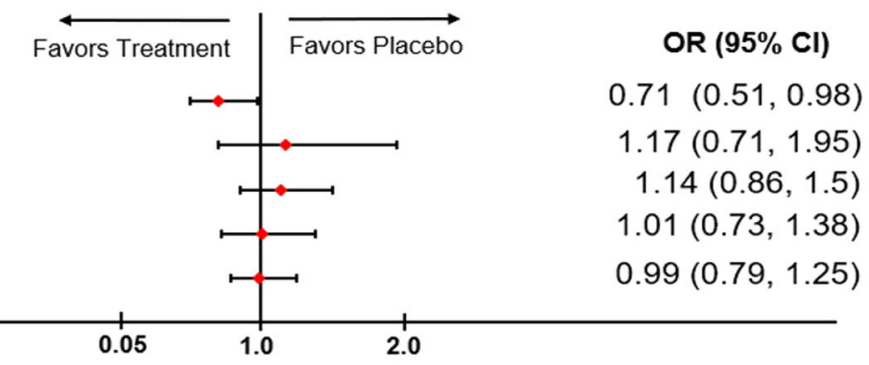

b

Discontinuation due to Lack of Efficacy

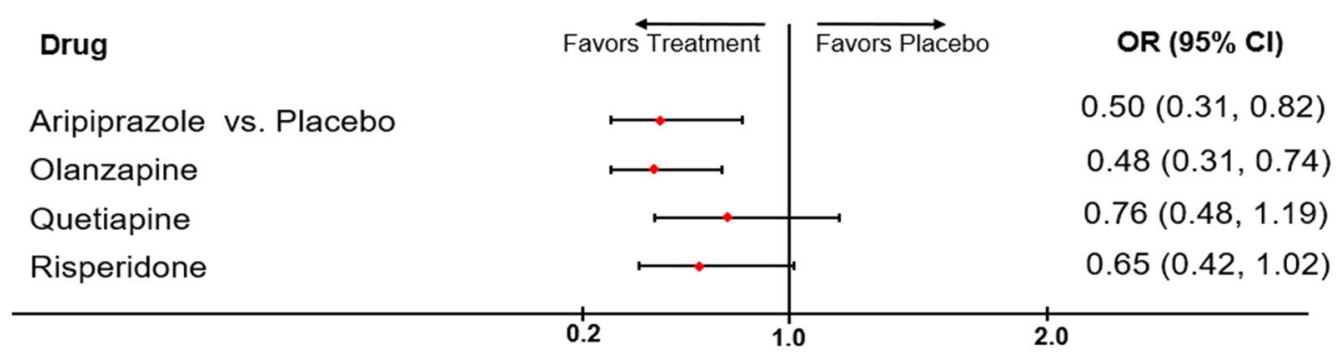

C Discontinuation due to Adverse Events

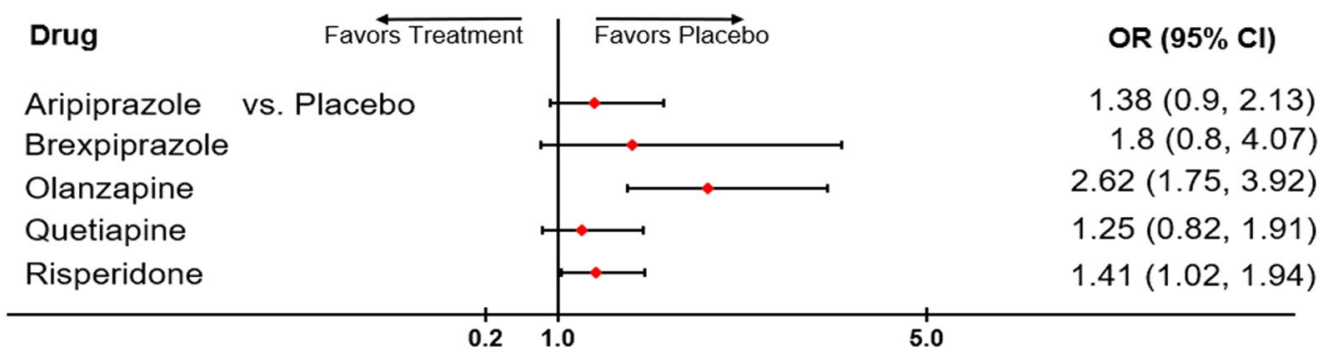

Fig. 4 Forest plots for acceptability outcomes of a typical antipsychotics compared with placebo. a Forest plot for all cause discontinuation, representing pooled odds ratio comparing each treatment option with placebo. The diamonds denote the point estimate for each treatment compared to placebo, while the horizontal line denoted the 95\% CI. If the 95\% CI does not cross 1 (null value); the results imply statistical significance. b Forest plot for discontinuation due to lack of efficacy, representing pooled odds ratio comparing each treatment option with placebo. The diamonds denote the point estimate for each

brexpiprazole (OR 0.40; 95\% CI 0.08, 2.12), followed by quetiapine (OR $0.77 ; 95 \%$ CI 0.48 , 1.24) and risperidone (OR 0.79; 95\% CI 0.63, 0.99) compared to placebo. Results for risperidone were significant. Odds were shown to be higher than placebo for aripiprazole and treatment compared to placebo, while the horizontal line denoted the $95 \%$ CI. If the $95 \%$ CI does not cross 1 (null value); the results imply statistical significance. c Forest plot for discontinuation due to adverse events, representing pooled odds ratio comparing each treatment option with placebo. The diamonds denote the point estimate for each treatment compared to placebo, while the horizontal line denoted the $95 \%$ CI. If the $95 \%$ CI does not cross 1 (null value); the results imply statistical significance

olanzapine (Supplementary Table 2). SUCRA results show that brexpiprazole $(84.4 \%)$ had the highest probability of being the safest for falls, fractures, and injuries. The SUCRA results for primary safety outcomes are reported in Supplementary Fig. 3. Additionally, results of all 
secondary outcomes in the analysis (cardiovascular AEs, EPS, akathisia) are reported in Supplementary Table 6 .

\section{Acceptability Outcomes}

Discontinuations: All Cause, Lack of Efficacy, and Adverse Events Supplementary Figure 2 includes the network diagram, and Fig. 4 depicts the forest plots for all acceptability outcomes. For the analysis of all-cause discontinuations, a total of 20 studies [31-36, 38-43, 45-51] $\quad(n=5744$ patients $)$ showed that odds of all-cause discontinuation were significantly lower for aripiprazole (OR $0.71 ; 95 \%$ CI $0.51,0.98$ ) compared to placebo. Results for all other AAPs were not statistically significant compared with each other. SUCRA results show that aripiprazole $(95.6 \%)$ had the highest probability of not being discontinued because of all causes.

For the analysis of discontinuations due to lack of efficacy, a total of 12 studies $[33-35,40,42,43,45,47-51] \quad(n=4382$ patients) showed that aripiprazole (OR 0.5; 95\% CI $0.31,0.82$ ) and olanzapine (OR 0.48; 95\% CI $0.31,0.74)$ had significantly lower odds of discontinuation while quetiapine (OR 0.76; 95\% CI $0.48,1,19)$ compared to placebo, while risperidone (OR 0.65; 95\% CI 0.42, 1.02) did not. SUCRA results show that olanzapine $(85.2 \%)$ had the highest probability of not being discontinued because of lack of efficacy.

For discontinuations due to adverse events, a total of 19 studies [31, 33-36, 39-51] $(n=5445$ patients) show that the odds of discontinuations were higher than placebo for olanzapine (OR 2.62; 95\% CI 1.75, 3.92), brexpiprazole (OR 1.80; 95\% CI 0.80, 4.07), quetiapine (OR 1.25; $95 \%$ CI $0.82,1.91$ ), aripiprazole (OR 1.38; 95\% CI 0.90, 2.13), and risperidone (OR 1.41; 95\% CI 1.02, 1.94) (Supplementary Table 3). SUCRA results show that placebo $(94.2 \%)$ had the highest probability of not being discontinued because of adverse events. Supplementary Fig. 3 report SUCRA diagram for acceptability outcomes.

\section{Other Outcomes}

MMSE, Cognitive Impairment Supplementary Figure 2 shows the network diagram for other outcomes. For the analysis of MMSE, a total of nine studies [33, 37, 41-43, 45, 46, 48, 50] $(n=1447)$ reported that aripiprazole (SMD $-0.09 ; 95 \%$ CI $-0.23,0.06$ ), quetiapine (SMD $-0.05 ; 95 \%$ CI $-0.26,0.16)$, and risperidone (SMD - 0.15; 95\% CI $(-0.39,0.09)$ demonstrated small numerical improvements in MMSE scale, while olanzapine (SMD 0.23; 95\% CI $-0.71,1.17$ ) did not show improvements compared to placebo.

For the analysis of cognitive impairment, a total of three studies [38, 41, 49] $(n=501$ patients) demonstrated that, compared to placebo, the odds of cognitive impairment were significantly higher for olanzapine (OR 7.29; 95\% CI 1.39, 38.24) (Supplementary Table 4).

\section{DISCUSSION}

This NMA evaluated the outcomes of AAPs in treating psychosis in patients with dementia and builds on a previous study by Yunusa et al. [11]. While this NMA assessed the efficacy, safety, and acceptability of AAPs in treating characteristic symptoms of DRP such as hallucinations and delusions, the previous study evaluated the efficacy and safety of AAPs on the broader gamut of NPS. Also, the previous study included only randomized, blinded clinical trials, while the current NMA included blinded and unblinded randomized and non-randomized trials and observational studies. Of the total 22 studies, 2 (Devanand, Ellingrod) were not RCTs. Given the small number, addition of these did not impact the results of the studied outcomes. Additionally, this study represents the most recent and comprehensive NMA of AAPs in DRP to date. Interestingly, this NMA confirms the previous findings that no single AAP showed better efficacy and safety results compared to another across all studied outcomes, and all AAPs were associated with an 
increased risk of adverse events. Furthermore, this study adds to the previous NMAs in the following ways: First, this study included brexpiprazole in addition to aripiprazole, olanzapine, quetiapine, and risperidone because the trial of brexpiprazole was published in 2020, after the previous NMA of Yunusa et al. was published. Of note, this NMA only used the safety and acceptability outcomes of brexpiprazole because the trial's efficacy outcome was based on agitation, measured using different domains on the NPS scale. Second, it evaluated the acceptability of AAPs in the form of treatment discontinuation outcomes. Given that treatment discontinuations are considered to reflect possible real-world effectiveness, it may provide a window into understanding the potential comparative effects of these treatments in routine clinical practice.

Our study results suggest that, compared with placebo, olanzapine demonstrated the greatest numerical non-significant symptom improvement on the NPI-NH psychosis subscale, followed by aripiprazole. On the other hand, quetiapine did not demonstrate numerical improvements compared with placebo. The previous NMA [11] reported all the AAPs showed small numerical improvements in the NPI total score compared to placebo, out of which aripiprazole had a significant improvement. Given that this study focused on the DRP population, we examined the NPI-NH psychosis subscale, which assessed the hallucination and delusion subset of the total NPI-NH questionnaire score. The difference in the outcomes measures between the two studies might explain why the acceptability of aripiprazole in this study was lower than what was reported by Yunusa et al. (SMD $=-0.2$ ).

Consistent with previously published NMAs and pairwise meta-analyses [10, 11, 52-55], this study affirmed that all existing AAPs have a greater risk of mortality compared to placebo. The previous study by Yunusa et al. reported risperidone to have the least risk of mortality, while this study showed that aripiprazole is associated with the least risk followed by risperidone, quetiapine, and olanzapine. This study found that brexpiprazole was associated with the highest risk of mortality. None of the
AAP-AAP comparisons for mortality outcome showed a statistically significant difference. The reported difference in the size of the odds of mortality with risperidone vs. placebo between Yunusa's previous research and the current study may be due to the differences in the number of studies as the previous study [11] included seven risperidone trials, while the current NMA included a total of eight risperidone studies. Nonetheless, the wide confidence intervals indicate a broad variability in estimates and suggest the need for more studies with a larger sample size. According to previously published NMA by Watts [10], APs had a higher risk of CVAEs compared to placebo. Consistent with other previous NMAs and pairwise meta-analyses, this study also reported that risperidone and olanzapine are significantly associated with CVAEs compared to placebo [9, 10, 52-55]. A fourfold increased risk of CVAEs was reported in this study, while the European Medical Agency and other studies reported that olanzapine has three times more risk of CVAEs compared to placebo [56]. Overall, according to current results, even though olanzapine showed improvements in efficacy, it demonstrated an alarming risk of mortality and CVAEs and should be avoided in patients with history of CVAEs or cardiovascular conditions. Like the previous findings of Yunusa et al. [11], olanzapine had the highest risk of FFI, followed by aripiprazole. In contrast, risperidone, quetiapine, and brexpiprazole had lower risk of FFI compared to placebo. Watt et al. reported APs to have lower odds of fractures but higher odds of falls compared to placebo. In this study, all AAPs had higher odds of somnolence compared to placebo. Quetiapine has the highest risk followed by olanzapine, aripiprazole, and risperidone, which was statistically significant and consistent with the previous findings [11]. It is plausible that quetiapine's sedative effect may be the reason for its frequent prescription potentially to induce sleep and temporarily calm symptoms [57, 58]. Olanzapine demonstrated a decrease in the MMSE scores as well as a potential increase in the cognitive impairment suggesting that cognitive decline may be accelerated with its use. Given the worsening of cognition associated with olanzapine from the 
two outcomes, they may need to be avoided in persons with moderate-to-severe DRP.

In alignment with the efficacy findings, the NMA results also demonstrated that olanzapine and aripiprazole are associated with lower odds of discontinuation due to lack of efficacy compared to placebo. Aripiprazole also demonstrated lower odds of all discontinuations. However, given their results on the safety outcomes, all the AAPs had high odds of discontinuation due to adverse events. All AAPs were associated with a high risk of mortality, CVAE, and somnolence, while risperidone showed significantly lower odds of FFIs. Regarding discontinuation of AAP as an acceptability measure, overall, no one drug gave lower odds of discontinuations due to all causes, lack of efficacy, and adverse reactions compared to placebo. Aripiprazole was the only drug that showed significantly better results compared to placebo for all-cause discontinuations. The lower odds of all-cause discontinuation with aripiprazole found in the study are not that surprising given that it is reported to be a relatively weight neutral antipsychotic, albeit with significant EPS liability such as akathisia [59]. Placebo comparisons showed that olanzapine and aripiprazole had significantly lower odds of discontinuation due to lack of efficacy followed by quetiapine and risperidone. These results are consistent with published evidence that consistently shows olanzapine to be a potent antipsychotic by improving neuropsychiatry scores, albeit with significant weight gain and other safety issues $[60,61]$. None of the studied AAPs demonstrated a better profile for discontinuation due to adverse events compared to placebo. In fact, results suggest that olanzapine demonstrated the highest odds of discontinuation due to $\mathrm{AE}$, which were significant, followed by brexpiprazole and risperidone. Due to lower adverse event outcomes, aripiprazole has the lowest odds for discontinuation due to AEs. Even though all AAPs are associated with some improvement in efficacy outcomes, they all are associated with some risk of AEs compared to placebo. Previously published literature suggested that discontinuation of AAP leads to worsening of symptoms in patients with greater baseline symptoms in the long run [62-64].
The high risk of AEs with AAPs reported in this NMA appears to support the American Geriatrics Society (AGS) Beers' criteria and APA guidelines regarding potentially inappropriate medication use with these off-label AAP use in older adults. While the Beers criteria suggest that AAP use should be avoided until all nonpharmacological treatment options have been exhausted or if patients are at risk of harm to self or to others [65], the APA guidelines suggest the use of non-pharmacological interventions prior to consideration of AAP use. The guidelines also recommend that treating physicians should assess the potential benefit and risks associated with the off-label AAPs and ensure that a shared decision-making about AAP treatment choice is made in discussion with patients or caregivers, as appropriate [66].

\section{Strengths and Limitations}

This NMA provides the most recent and comprehensive assessment of the comparative effects of the commonly used off-label AAPs. The NMA included more studies than previously published meta-analyses and was also intended as a comparative assessment of individual AAPs rather than an evaluation of the AAP class of drugs. This study was also the first to evaluate the acceptability of AAPs for the DRP population based on discontinuations. Remaining consistent with comparative efficacy and safety results reported here and elsewhere, the acceptability results demonstrate that no single AAP was more acceptable than any other or placebo on all three measures of discontinuations (all cause, lack of efficacy, and adverse events).

Despite its strengths, this NMA also has some limitations. Only articles that were published in the English language were included in the study, which may have introduced a language bias [67]. However, studies have shown that excluding articles not published in English language has little impact on summary treatment effect estimates [68, 69]. The study included a total of 22 studies, which led to small sample sizes for individual drug comparisons and consequently wider $95 \%$ CIs for some 
estimates. It is also plausible that inclusion of non-RCTs in the analyses may have affected the results. We were unable to provide evidence on time to death because of the paucity of information from studies included in this NMA. Despite these limitations, this study may help recognize the comparative effects of commonly used off-label AAPs and their individual benefitrisk profiles as it relates to treating the elderly population with DRP.

\section{CONCLUSIONS}

Overall results demonstrate that, compared with placebo, quetiapine is not associated with improvement in psychosis in patients with dementia, while olanzapine and aripiprazole show small numerical improvements that are not significant. These off-label AAPs (quetiapine, risperidone, olanzapine, aripiprazole, and brexpiprazole) are associated with greater odds of mortality, CVAEs, and discontinuations due to adverse events than placebo. The results suggest an existing gap in clinical decisionmaking given the need to systematically consider the AAP benefit-risk profile in making appropriate treatment selection among patients with DRP. Furthermore, these results underscore the ongoing unmet need for AAP treatment options with a more favorable benefit-risk profile for the treatment of DRP among patients with underlying dementia.

\section{ACKNOWLEDGEMENTS}

Funding. The research was funded by Acadia Pharmaceuticals Inc. Employees of Acadia Pharmaceuticals Inc. were a part of the research team for development of concept, study design, study selection, and interpretation of the results. The Journal's Rapid Service and Open Access Fees were also funded by Acadia Pharmaceuticals Inc.

Authorship. All named authors meet the International Committee of Medical Journal Editors (ICMJE) criteria for authorship for this article, take responsibility for the integrity of the work, and have given their approval for this version to be published.

Authors' Contributions. IY, NR, VA, GD, and $\mathrm{KR}$ developed the concept and study design; IY, NR, VA, GD, BM, and KR worked on study selection and interpretation of data; IY, $\mathrm{NR}, \mathrm{VA}, \mathrm{GD}, \mathrm{BM}$, and KR worked on preparation of the manuscript. All authors critically reviewed and approved the final version of the paper.

Disclosures. Nazia Rashid, George Demos, and Victor Abler are employees of Acadia Pharmaceuticals, Inc., which has funded the research. At the time of this research, George Demos was an employee of Acadia Pharmaceuticals, Inc., and is currently affiliated with Arrowhead Pharmaceuticals. Bhargavi Mahadik and Krithika Rajagopalan are employees of Anlitiks, Inc. Ismaeel Yunusa is affiliated as Assistant Professor at the University of South Carolina, College of Pharmacy, and is a former employee of Anlitiks, Inc. The authors confirm that funding has in no way influenced the outcome. There are no other conflicts of interest associated with this publication.

Compliance with Ethics Guidelines. This article is based on previously conducted studies and does not contain any new studies with human participants or animals performed by any of the authors.

Data Availability. The authors confirm that the data supporting the findings of this study are available within the article and its supplementary materials.

Open Access. This article is licensed under a Creative Commons Attribution-NonCommercial 4.0 International License, which permits any non-commercial use, sharing, adaptation, distribution and reproduction in any medium or format, as long as you give appropriate credit to the original author(s) and the source, provide a link to the Creative Commons licence, and indicate if changes were made. The images or other third party material in this article are 
included in the article's Creative Commons licence, unless indicated otherwise in a credit line to the material. If material is not included in the article's Creative Commons licence and your intended use is not permitted by statutory regulation or exceeds the permitted use, you will need to obtain permission directly from the copyright holder. To view a copy of this licence, visit http://creativecommons.org/licenses/by$\mathrm{nc} / 4.0 /$.

\section{REFERENCES}

1. Alzheimer's disease facts and figures. Alzheimers Dement. 2021;17(3):327-406.

2. Prince M, Ali GC, Guerchet M, Prina AM, Albanese $\mathrm{E}, \mathrm{Wu}$ YT. Recent global trends in the prevalence and incidence of dementia, and survival with dementia. Alzheimers Res Ther. 2016;8(1):23.

3. Kales HC, Gitlin LN, Lyketsos CG. Management of neuropsychiatric symptoms of dementia in clinical settings: recommendations from a multidisciplinary expert panel. J Am Geriatr Soc. 2014;62(4): 762-9.

4. Small GW. Managing the burden of dementia related delusions and hallucinations. J Fam Pract. 2020;69(7 Suppl):S39-44.

5. Sink KM, Holden KF, Yaffe K. Pharmacological treatment of neuropsychiatric symptoms of dementia: a review of the evidence. JAMA. 2005;293(5):596-608.

6. Steinberg M, Lyketsos CG. Atypical antipsychotic use in patients with dementia: managing safety concerns. Am J Psychiatry. 2012;169(9):900-6.

7. Maust DT, Kim HM, Seyfried LS, et al. Antipsychotics, other psychotropics, and the risk of death in patients with dementia: number needed to harm. JAMA Psychiat. 2015;72(5):438-45.

8. Citrome L, Eramo A, Francois C, et al. Lack of tolerable treatment options for patients with schizophrenia. Neuropsychiatr Dis Treat. 2015;11: 3095-104.

9. Mittal V, Kurup L, Williamson D, Muralee S, Tampi RR. Risk of cerebrovascular adverse events and death in elderly patients with dementia when treated with antipsychotic medications: a literature review of evidence. Am J Alzheimers Dis Other Demen. 2011;26(1):10-28.
10. Watt JA, Goodarzi Z, Veroniki AA, et al. Safety of pharmacologic interventions for neuropsychiatric symptoms in dementia: a systematic review and network meta-analysis. BMC Geriatr. 2020;20(1): 212.

11. Yunusa I, Alsumali A, Garba AE, Regestein QR, Eguale T. Assessment of reported comparative effectiveness and safety of atypical antipsychotics in the treatment of behavioral and psychological symptoms of dementia: a network meta-analysis. JAMA Netw Open. 2019;2(3): e190828.

12. Yunusa I, Rashid N, Abler V, Rajagopalan K. Comparative efficacy, safety, tolerability, and effectiveness of antipsychotics in the treatment of dementia-related psychosis (DRP): a systematic literature review. J Prev Alzheimer's Dis. 2021;8: 520-33.

13. Moher D, Liberati A, Tetzlaff J, Altman DG, Group P. Preferred reporting items for systematic reviews and meta-analyses: the PRISMA statement. BMJ. 2009;339:b2535.

14. Reviews UoYNCf, Dissemination. Systematic Reviews: CRD's Guidance for Undertaking Reviews in Health Care. Centre for Reviews and Dissemination; 2009

15. Higgins JP, Altman DG, Gotzsche PC, et al. The Cochrane Collaboration's tool for assessing risk of bias in randomised trials. BMJ. 2011;343:d5928.

16. Wells GA, Wells G, Shea B, et al. The NewcastleOttawa Scale (NOS) for assessing the quality of nonrandomised studies in meta-analyses. 2014

17. Hutton B, Salanti G, Caldwell DM, et al. The PRISMA extension statement for reporting of systematic reviews incorporating network meta-analyses of health care interventions: checklist and explanations. Ann Intern Med. 2015;162(11): 777-84.

18. Riley RD, Jackson D, Salanti G, et al. Multivariate and network meta-analysis of multiple outcomes and multiple treatments: rationale, concepts, and examples. BMJ. 2017;358: j3932.

19. Shim S, Yoon BH, Shin IS, Jong-Myon B. Network meta-analysis: application and practice using Stata. Epidemiol Health. 2017;39: e2017047.

20. Salanti G. Indirect and mixed-treatment comparison, network, or multiple-treatments meta-analysis: many names, many benefits, many concerns for the next generation evidence synthesis tool. Res Synth Methods. 2012;3(2):80-97.

21. Jansen JP, Naci H. Is network meta-analysis as valid as standard pairwise meta-analysis? It all depends 
on the distribution of effect modifiers. BMC Med. 2013;11:159.

22. Bucher HC, Guyatt GH, Griffith LE, Walter SD. The results of direct and indirect treatment comparisons in meta-analysis of randomized controlled trials. J Clin Epidemiol. 1997;50(6):683-91.

23. Chaimani A, Salanti G. Visualizing assumptions and results in network meta-analysis: the network graphs package. Stand Genom Sci. 2015;15(4): 905-50.

24. Dias S, Welton NJ, Caldwell DM, Ades AE. Checking consistency in mixed treatment comparison metaanalysis. Stat Med. 2010;29(7-8):932-44.

25. Higgins JP, Jackson D, Barrett JK, Lu G, Ades AE, White IR. Consistency and inconsistency in network meta-analysis: concepts and models for multiarm studies. Res Synth Methods. 2012;3(2):98-110.

26. Tonin FS, Rotta I, Mendes AM, Pontarolo R. Network meta-analysis: a technique to gather evidence from direct and indirect comparisons. Pharm Pract (Granada). 2017;15(1):943.

27. van Valkenhoef $G$, Dias $S$, Ades AE, Welton NJ. Automated generation of node-splitting models for assessment of inconsistency in network meta-analysis. Res Synth Methods. 2016;7(1):80-93.

28. Faraone SV. Interpreting estimates of treatment effects: implications for managed care. $\mathrm{P} T$. 2008;33(12):700-11.

29. Salanti G, Ades AE, Ioannidis JP. Graphical methods and numerical summaries for presenting results from multiple-treatment meta-analysis: an overview and tutorial. J Clin Epidemiol. 2011;64(2): 163-71.

30. Veroniki AA, Straus SE, Rucker G, Tricco AC. Is providing uncertainty intervals in treatment ranking helpful in a network meta-analysis? J Clin Epidemiol. 2018;100:122-9.

31. Ballard C, Margallo-Lana M, Juszczak E, et al. Quetiapine and rivastigmine and cognitive decline in Alzheimer's disease: randomised double blind placebo controlled trial. BMJ. 2005;330(7496):874.

32. Brodaty $\mathrm{H}$, Ames D, Snowdon J, et al. A randomized placebo-controlled trial of risperidone for the treatment of aggression, agitation, and psychosis of dementia. J Clin Psychiatry. 2003;64:134-43.

33. De Deyn P, Jeste DV, Swanink R, et al. Aripiprazole for the treatment of psychosis in patients with Alzheimer's disease: a randomized, placebo-controlled study. J Clin Psychopharmacol. 2005;25(5): 463-7.
34. De Deyn PP, Carrasco MM, Deberdt W, et al. Olanzapine versus placebo in the treatment of psychosis with or without associated behavioral disturbances in patients with Alzheimer's disease. Int J Geriatr Psychiatry. 2004;19(2):115-26.

35. Deberdt WG, Dysken MW, Rappaport SA, et al. Comparison of olanzapine and risperidone in the treatment of psychosis and associated behavioral disturbances in patients with dementia. Am J Geriatr Psychiatry. 2005;13(8):722-30.

36. Devanand D, Mintzer J, Schultz SK, et al. Relapse risk after discontinuation of risperidone in Alzheimer's disease. N Engl J Med. 2012;367(16): 1497-507.

37. Ellingrod VL, Schultz SK, Ekstam-Smith K, Kutscher E, Turvey C, Arndt S. Comparison of risperidone with olanzapine in elderly patients with dementia and psychosis. Pharmacotherapy. 2002;22(1):1-5.

38. Gareri P, Cotroneo A, Lacava R, et al. Comparison of the efficacy of new and conventional antipsychotic drugs in the treatment of behavioral and psychological symptoms of dementia (BPSD). Arch Gerontol Geriatr Suppl. 2004;9:207.

39. Grossberg GT, Kohegyi E, Mergel V, et al. Efficacy and safety of brexpiprazole for the treatment of agitation in Alzheimer's dementia: two 12-week, randomized, double-blind, placebo-controlled trials. Am J Geriatr Psychiatry. 2020;28(4):383-400.

40. Katz IR, Jeste DV, Mintzer JE, Clyde C, Napolitano J, Brecher M. Comparison of risperidone and placebo for psychosis and behavioral disturbances associated with dementia: a randomized, double-blind trial. Risperidone Study Group. J Clin Psychiatry. 1999;60(2):107-15.

41. Kurlan R, Cummings J, Raman R, Thal L. Quetiapine for agitation or psychosis in patients with dementia and parkinsonism. Neurology. 2007;68(17):1356-63.

42. Mintzer J, Greenspan A, Caers I, et al. Risperidone in the treatment of psychosis of Alzheimer disease: results from a prospective clinical trial. Am J Geriatr Psychiatry. 2006;14(3):280-91.

43. Mintzer JE, Tune LE, Breder CD, et al. Aripiprazole for the treatment of psychoses in institutionalized patients with Alzheimer dementia: a multicenter, randomized, double-blind, placebo-controlled assessment of three fixed doses. Am J Geriatr Psychiatry. 2007;15(11):918-31.

44. Mulsant BH, Gharabawi GM, Bossie CA, et al. Correlates of anticholinergic activity in patients with dementia and psychosis treated with risperidone or olanzapine. J Clin Psychiatry. 2004;65(12):1708-14. 
45. Paleacu D, Barak Y, Mirecky I, Mazeh D. Quetiapine treatment for behavioural and psychological symptoms of dementia in Alzheimer's disease patients: a 6-week, double-blind, placebo-controlled study. Int J Geriatr Psychiatry. 2008;23(4): 393-400.

46. Rainer M, Haushofer M, Pfolz H, Struhal C, Wick W. Quetiapine versus risperidone in elderly patients with behavioural and psychological symptoms of dementia: efficacy, safety and cognitive function. Eur Psychiatry. 2007;22(6):395-403.

47. Street JS, Clark WS, Gannon KS, et al. Olanzapine treatment of psychotic and behavioral symptoms in patients with Alzheimer disease in nursing care facilities: a double-blind, randomized, placebocontrolled trial. Arch Gen Psychiatry. 2000;57(10): 968-76.

48. Streim JE, Porsteinsson AP, Breder CD, et al. A randomized, double-blind, placebo-controlled study of aripiprazole for the treatment of psychosis in nursing home patients with Alzheimer disease. Am J Geriatr Psychiatry. 2008;16(7):537-50.

49. Sultzer DL, Davis SM, Tariot PN, et al. Clinical symptom responses to atypical antipsychotic medications in Alzheimer's disease: phase 1 outcomes from the CATIE-AD effectiveness trial. Am J Psychiatry. 2008;165(7):844-54.

50. Tariot PN, Schneider L, Katz IR, et al. Quetiapine treatment of psychosis associated with dementia: a double-blind, randomized, placebo-controlled clinical trial. Am J Geriatr Psychiatry. 2006;14(9): 767-76.

51. Zhong KX, Tariot P, Mintzer J, Minkwitz M, Devine N. Quetiapine to treat agitation in dementia: a randomized, double-blind, placebo-controlled study. Curr Alzheimer Res. 2007;4(1):81-93.

52. Jin B, Liu H. Comparative efficacy and safety of therapy for the behavioral and psychological symptoms of dementia: a systemic review and Bayesian network meta-analysis. J Neurol. 2019;266(10):2363-75.

53. Maher AR, Maglione M, Bagley S, et al. Efficacy and comparative effectiveness of atypical antipsychotic medications for off-label uses in adults: a systematic review and meta-analysis. JAMA. 2011;306(12): 1359-69.

54. Schneider LS, Dagerman K, Insel PS. Efficacy and adverse effects of atypical antipsychotics for dementia: meta-analysis of randomized, placebocontrolled trials. Am J Geriatr Psychiatry. 2006;14(3):191-210.
55. Schneider LS, Dagerman KS, Insel P. Risk of death with atypical antipsychotic drug treatment for dementia: meta-analysis of randomized placebocontrolled trials. JAMA. 2005;294(15):1934-43.

56. EMEA public statement on the safety of olanzapine. http://www.bfarm.de/SharedDocs/Downloads/DE/ Arzneimittel/Pharmakovigilanz/Service/aktuelles/ emea-zyprexa-st.pdf?_blob=publicationFile\&v=2 . Published 2004. Accessed 13 Jan 2021.

57. Dolder CR, McKinsey J. Quetiapine for sleep in patients with dementia. Consult Pharm. 2010;25(10):676-9.

58. Nerius M, Johnell K, Garcia-Ptacek S, Eriksdotter M, Haenisch B, Doblhammer G. The impact of antipsychotic drugs on long-term care, nursing home admission, and death in dementia patients. J Gerontol A Biol Sci Med Sci. 2018;73(10): 1396-402.

59. Lindsey RL, Kaplan D, Koliatsos V, Walters JK, Sandson NB. Aripiprazole and extrapyramidal symptoms. J Am Acad Child Adolesc Psychiatry. 2003;42(11):1268-9.

60. Allison DB, Casey DE. Antipsychotic-induced weight gain: a review of the literature. J Clin Psychiatry. 2001;62(Suppl 7):22-31.

61. Rocca P, Marino F, Montemagni C, Perrone D, Bogetto F. Risperidone, olanzapine and quetiapine in the treatment of behavioral and psychological symptoms in patients with Alzheimer's disease: preliminary findings from a naturalistic, retrospective study. Psychiatry Clin Neurosci. 2007;61(6): 622-9.

62. Declercq T, Petrovic M, Azermai M, et al. Withdrawal versus continuation of chronic antipsychotic drugs for behavioural and psychological symptoms in older people with dementia. Cochrane Database Syst Rev. 2013;3:CD007726.

63. Pan YJ, Wu CS, Gau SS, Chan HY, Banerjee S. Antipsychotic discontinuation in patients with dementia: a systematic review and meta-analysis of published randomized controlled studies. Dement Geriatr Cogn Disord. 2014;37(3-4):125-40.

64. Tampi RR, Tampi DJ, Balachandran S, Srinivasan S. Antipsychotic use in dementia: a systematic review of benefits and risks from meta-analyses. Ther Adv Chronic Dis. 2016;7(5):229-45.

65. By the American Geriatrics Society Beers Criteria Update Expert P. American Geriatrics Society 2015 Updated Beers Criteria for Potentially Inappropriate Medication Use in Older Adults. J Am Geriatr Soc. 2015;63(11):2227-2246. 
66. Reus VI, Fochtmann LJ, Eyler AE, et al. The American Psychiatric Association practice guideline on the use of antipsychotics to treat agitation or psychosis in patients with dementia. Am J Psychiatry. 2016;173(5):543-6.

67. Boutron I, Page MJ, Higgins JP, et al. Considering bias and conflicts of interest among the included studies. In: Cochrane Handbook for Systematic Reviews of Interventions. pp. 177-204. 2019.
68. Morrison A, Polisena J, Husereau D, et al. The effect of English-language restriction on systematic review-based meta-analyses: a systematic review of empirical studies. Int J Technol Assess Health Care. 2012;28(2):138-44.

69. Juni P, Holenstein F, Sterne J, Bartlett C, Egger M. Direction and impact of language bias in metaanalyses of controlled trials: empirical study. Int J Epidemiol. 2002;31(1):115-23. 